\title{
Philosophieren mit Kindern
}

\section{Barbara Brüning}

Eva Zoller Morf: Selber denken macht schlau. Philosophieren mit Kindern und Jugendlichen. Oberhofen: Zytglogge 2010.

1 Eva Zoller Morf leitet seit 1987 die von ihr gegründete Schweizerische Dokumentationsstelle für Kinder- und Alltagsphilosophie. Sie legt mit ihrem dritten Buch ein Kompendium ihrer mehr als 2ojährigen praktischen Tätigkeit vor, die hauptsächlich Kurse für Kinder, Eltern und Erzieherinnen im Bereich des Philosophierens mit Kindern umfasst sowie Dozenten?tätigkeit an Pädagogischen Hochschulen in der Schweiz.

Im ersten Teil ihres Buches thematisiert die Autorin den Unterschied zwischen kleinen und großen (philosophischen) Kinderfragen und gibt Tipps, wie Erwachsene damit umgehen können (S. 30). Ein neuer Aspekt ist hierbei die ?Werkzeugkiste des schlauen Denkers?, die auf dem didaktischen Modell des hawaiianischen Philosophieprofessors Thomas E. Jackson basiert, mit dem Eva Zoller in den letzten Jahren zusammengearbeitet hat.

Die Werkzeugkiste umfasst sieben Werkzeuge: die Überprüfung der eigenen Meinung (Stimmt das wirklich so?), Beispiele und Gegenbeispiele für Meinungen finden, begriffliche Präzisierungen geben, nach Begründungen suchen sowie Annahmen und Schlussfolgerungen treffen. Den Gebrauch dieser Werkzeuge stellt die Autorin anhand von verschiedenen Kinderbüchern für das Vor- und Grundschulalter vor.

Im zweiten Teil des Buches steht das Philosophieren über Werte, Wünsche und Gefühle mit Grundschulkindern im Mittelpunkt. Eva Zoller widmet dabei dem von der kürzlich verstor-benen amerikanischen Philosophin Ann Margret Sharp fokussierten Caring Thinking (fürsorglich teilnehmendes Denken) große Aufmerksamkeit (S. 66-70). Das Philosophieren mit Kindern soll nicht nur auf die Entwicklung von Denkfähigkeiten abzielen, sondern auch die Emo?tionalität der Kinder wie Einfühlungsvermögen, Mitgefühl und Gerechtigkeitssinn entwic?keln. Seine Bestandteile umfassen wertschätzendes Denken (moralische Sensibilität für Naturereignisse, menschliches Verhalten, Kulturen), affektives Denken (Empathie und Partizipation an Projekten), aktives Denken (Problemlösungskompetenz) sowie normatives Denken (Vergleich von Ist-Zustand und SollZustand). Alle vier Denkformen tragen nach Ansicht von Eva Zoller Morf dazu bei, Kinder anzuregen, nach eigenen Antworten auf wichtige Sinnfragen zu suchen. In diesem Zusammenhang sollten Kinder auch lernen, eine Sprache für ihre Gefühle zu finden.

Die gegenwärtige Diskussion in der Bundesrepublik Deutschland über das Zusammenleben von Menschen unterschiedlicher Kulturen und Religionen verleiht dem Caring Thinking eine zunehmende Bedeutung ? es sollte für die Didaktik des Ethik- und Philosophieunterrichts stärker elaboriert werden.

Der dritte Teil des Buches beschäftigt sich mit existentiellen Fragen nach Wahrheit, Gott und Tod. Hierzu werden Beispiele praktischen Arbeitens vorgestellt, die sich vorwiegend auf die Klassen 5/6 beziehen. Eva Zoller Morf zeigt beispielsweise Möglichkeiten zur Begriffsarbeit über Seele und Geist (S. 126), sie stellt Gesprächsanregungen (Hebammenfragen) zu den Themen Wahrheit sowie Leben und Tod vor und erklärt anhand des Fünf-Finger-Modells von Ekkehard Martens (die phänomenologische, hermeneutische, dialektische, analytische und spekulative Methode), wie mit Jugendlichen über Identität philosophiert werden kann (S. 133/34).

Das Buch von Eva Zoller Morf gibt eine anschauliche Zusammenfassung verschiedener aktueller Tendenzen zum Philosophieren mit Kindern, die sich im Wesentlichen an neueren Entwicklungen in den USA orientieren. Es eröffnet vielfältige Möglichkeiten des außerschulischen und fächerübergreifenden Philosophierens im Vor- und Grundschulalter und präsentiert eine Schatzkiste von Kinderbüchern zu den großen Fragen menschlichen Daseins.

1. Abdruck von: Zeitschrift für Didaktik der Philosophie und Ethik, case Nr. 1/2011, storyJahrgang 33, Februar 2011 (c) by ZDPE 2011. (context) 